\title{
Pancreatic follicular lymphoma: a report of two cases and literature review
}

\author{
Takeshi Okamoto $^{1} \cdot$ Takashi Sasaki $^{1}$ (1) $\cdot$ Noriko Nishimura $^{2} \cdot$ Manabu Takamatsu $^{3} \cdot$ Chinatsu Mori $^{1} \cdot$ Takafumi Mie $^{1}$. \\ Takaaki Furukawa ${ }^{1}$. Yuto Yamada ${ }^{1}$ Tsuyoshi Takeda ${ }^{1}$. Akiyoshi Kasuga ${ }^{1} \cdot$ Masato Matsuyama ${ }^{1} \cdot$ Masato Ozaka $^{1}$. \\ Dai Maruyama ${ }^{2} \cdot$ Naoki Sasahira $^{1}$
}

Received: 19 July 2021 / Accepted: 23 August 2021 / Published online: 27 August 2021

(c) Japanese Society of Gastroenterology 2021

\begin{abstract}
Primary pancreatic lymphomas are extremely rare, accounting for $0.1-0.5 \%$ of malignant lymphomas and about $0.2 \%$ of primary pancreatic tumors. They occur most commonly in the pancreatic head of elderly males, with diffuse large B-cell lymphoma being the most common subtype. Primary pancreatic follicular lymphoma is the most commonly reported primary pancreatic indolent lymphoma, accounting for $9-14 \%$ of primary pancreatic lymphomas. We report two cases of primary pancreatic follicular lymphoma treated with obinutuzumab, a second-generation humanized anti-CD20 monoclonal antibody, and bendamustine. One was diagnosed by endoscopic ultrasound-guided fine-needle aspiration, while the other required laparoscopic lymph node sampling to reach a diagnosis. Both achieved complete response with induction therapy and opted for maintenance therapy with obinutuzumab. We also conducted a literature review of primary pancreatic follicular lymphoma cases reported over the last 30 years.
\end{abstract}

Keywords Malignant lymphoma · Endoscopic ultrasound-guided fine-needle aspiration · Monoclonal antibody $\cdot$ Pancreas

\section{Introduction}

Primary pancreatic lymphomas (PPL) are extremely rare, accounting for $0.1-0.5 \%$ of malignant lymphomas and about $0.2 \%$ of primary pancreatic tumors [1-3]. They occur most commonly in the pancreatic head of elderly males, with diffuse large B-cell lymphoma being the most common subtype [4]. The diagnosis of PPL can be made if lymphoma is mainly localized in the pancreas, even if there is peripheral lymph node involvement or distant spread [5]. Primary pancreatic follicular lymphoma (PPFL) is the most commonly

Takashi Sasaki

sasakit-tky@umin.ac.jp

1 Department of Hepato-Biliary-Pancreatic Medicine, Cancer Institute Hospital of Japanese Foundation for Cancer Research, 3-8-31 Ariake, Koto-ku, Tokyo 135-8550, Japan

2 Department of Hematology Oncology, Cancer Institute Hospital of Japanese Foundation for Cancer Research, 3-8-31 Ariake, Koto-ku, Tokyo 135-8550, Japan

3 Department of Pathology, Cancer Institute Hospital of Japanese Foundation for Cancer Research, 3-8-31 Ariake, Koto-ku, Tokyo 135-8550, Japan reported indolent PPL, with up to 31 reports over the last 30 years [2, 4, 6-18]. PPFL accounts for 9-14\% of PPLs [17-19].

PPFL presents difficulties in diagnosis. The differential diagnosis may include neuroendocrine neoplasms, autoimmune pancreatitis and, most importantly, pancreatic ductal adenocarcinoma [14, 16]. Diagnostic accuracy of endoscopic ultrasound-guided fine-needle aspiration (EUS-FNA) in PPFL may be suboptimal [11]. It is therefore not unusual for PPFLs to be diagnosed only after surgical resection [15, $16,18]$.

As treatment of follicular lymphoma is not dictated by origin, standard regimens can be used for extranodal disease, including PPFL. Treatment outcomes in PPFL have not been elucidated, owing to its rarity. While obinutuzumab-based regimens have come to be accepted as first-line therapy in advanced follicular lymphoma with high tumor burden [20], its safety and efficacy in PPFL have not been reported. We report two cases of successful diagnosis and treatment of PPFL and review the English literature on PPFL. 


\section{Case reports}

\section{Case 1}

An asymptomatic 67-year-old man was referred for multiple masses in the pancreatic head discovered on screening abdominal ultrasound. He was also receiving treatment for hypertension and dyslipidemia, which were wellcontrolled with medications. He had no family history of malignancies. Physical examination was unremarkable. Laboratory tests revealed an increase in soluble interleukin-2 receptor to $623 \mathrm{U} / \mathrm{mL}$ (reference range 145-519 $\mathrm{U} / \mathrm{mL}$ ); all other results including immunoglobulin $\mathrm{G} 4$ (IgG4), carcinoembryonic antigen (CEA) and carbohydrate antigen 19-9 (CA 19-9) were within the normal range. The hepatitis $\mathrm{B}$ panel was negative.

Computed tomography (CT) with contrast revealed a slowly-enhancing $4 \mathrm{~cm}$ mass in the pancreatic head. Multiple mildly enlarged lymph nodes in the hepatoduodenal ligament and mesentery were also observed, with no clear vascular invasion. There was no biliary or pancreatic duct dilatation or signs of chronic pancreatitis (Fig. 1a). Magnetic resonance imaging (MRI) with contrast revealed a heterogeneous pancreatic mass and mesenteric lymphadenopathy with abnormal diffusion restriction. $18 \mathrm{~F}$-fluorodeoxyglucose positron emission tomography (FDG-PET)/CT showed increased uptake in the pancreatic head (maximum Standardized Uptake Value (SUVmax): 6.7) and para-aortic lymph nodes (SUVmax: 2.9) (Fig. 1b). A mass with SUVmax of 9.1 was also noted in the right lobe of thyroid.

EUS revealed a $36 \times 34 \mathrm{~mm}$ heterogeneous, multi-nodular mass in the pancreatic head near the inferior duodenal angle (Fig. 1c). Borders were relatively clearly delineated. Significant blood flow was confirmed on Doppler EUS. Contrast-enhanced EUS showed early enhancement similar to the adjacent normal pancreas, which was not typical for adenocarcinoma but not as strongly enhanced as may be expected for neuroendocrine neoplasms or autoimmune pancreatitis. Multiple slightly enlarged lymph nodes were also noted. IgG4-related pancreatitis was believed to be the leading differential, while pancreatic cancer and malignant lymphoma could not be ruled out.

EUS-FNA was performed using a 22-gauge fine-needle biopsy needle (Acquire, Boston Scientific, Marlborough, MA, USA). Two passes were made using the slow-pull technique with 20 strokes per pass, achieving an adequate specimen for pathological diagnosis. Histological evaluation of the specimen revealed a dense, monotonous proliferation of atypical lymphocytes. Immunohistochemistry was consistent with Grade 1-2 follicular lymphoma: CD5-, $\mathrm{CD} 10+, \mathrm{CD} 20+$. BCL2 +, BCL6 +, MUM1- (Fig. 2). Ki-67 labeling index was less than $1 \%$, indicating low proliferative activity. Only a few plasma cells were positive for IgG4 staining.

Esophagogastroduodenoscopy and colonoscopy were unremarkable. Bone marrow aspiration was negative for malignancy. As a result, the patient was diagnosed with PPFL with low tumor burden, Grade 1-2, Lugano classification stage IIE, with a Follicular Lymphoma International Prognostic Index (FLIPI) of 1 (age): low risk, assuming that the thyroidal lesion was not lymphoma. If the thyroid was involved, the diagnosis would be Lugano classification stage IIIE, with a FLIPI of 2 (age, stage): intermediate risk.

Despite the low tumor volume, the multiple pancreatic masses posed a high risk of biliary obstruction. There was also a possibility that the lymphoma was already at an advanced stage, as it could not be determined at the time whether the thyroid was involved or not. Informed consent was therefore obtained for treatment with obinutuzumab and bendamustine (GB) Six cycles of GB led to complete response with minimal adverse effects. As the thyroid mass remained unchanged, we determined that it was a separate clinical entity from the lymphoma. Surgical resection was
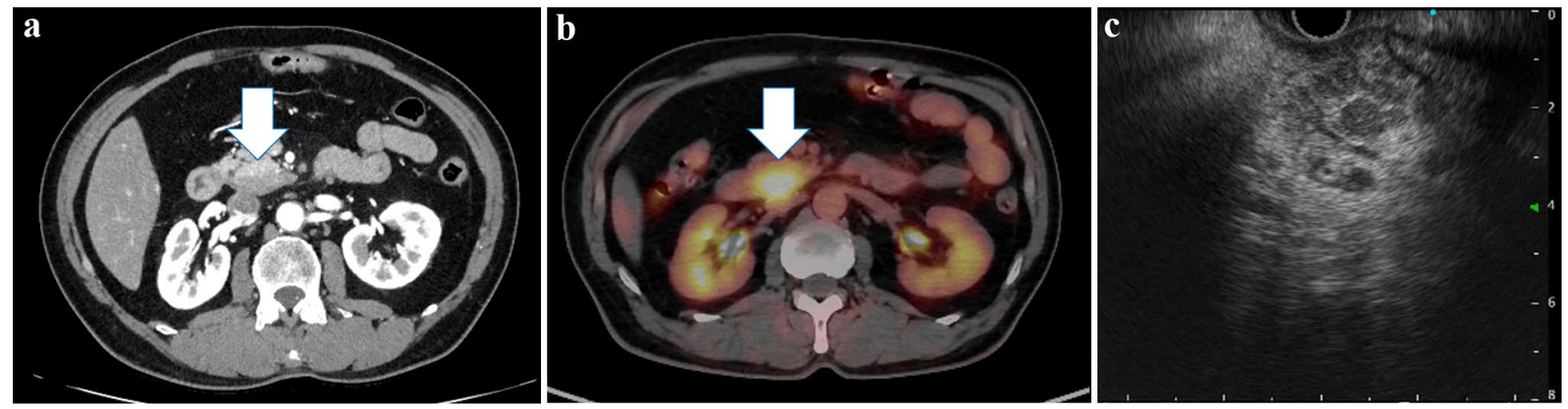

Fig. 1 a Computed tomography (CT) with contrast revealed a slowlyenhancing $4 \mathrm{~cm}$ mass in the pancreatic head (arrow). b 18F-fluorodeoxyglucose positron emission tomography/CT revealed increased uptake (arrow) in the pancreatic head (maximum Standardized
Uptake Value: 6.7). c Endoscopic ultrasound revealed a $36 \times 34 \mathrm{~mm}$ clearly delineated, heterogeneous, multi-nodular mass in the pancreatic head 
Fig. 2 Histological evaluation of the endoscopic ultrasoundguided fine-needle aspiration specimen. a Hematoxylin and eosin staining with low-power (scale bar: $200 \mu \mathrm{m}$ ) and b high-power (scale bar: $100 \mu \mathrm{m}$ ) magnifications revealed a dense, monotonous proliferation of atypical lymphocytes. Immunohistochemistry was positive for c CD20, d BCL2, e BCL6, and f CD10 (scale bars: $100 \mu \mathrm{m}$ )
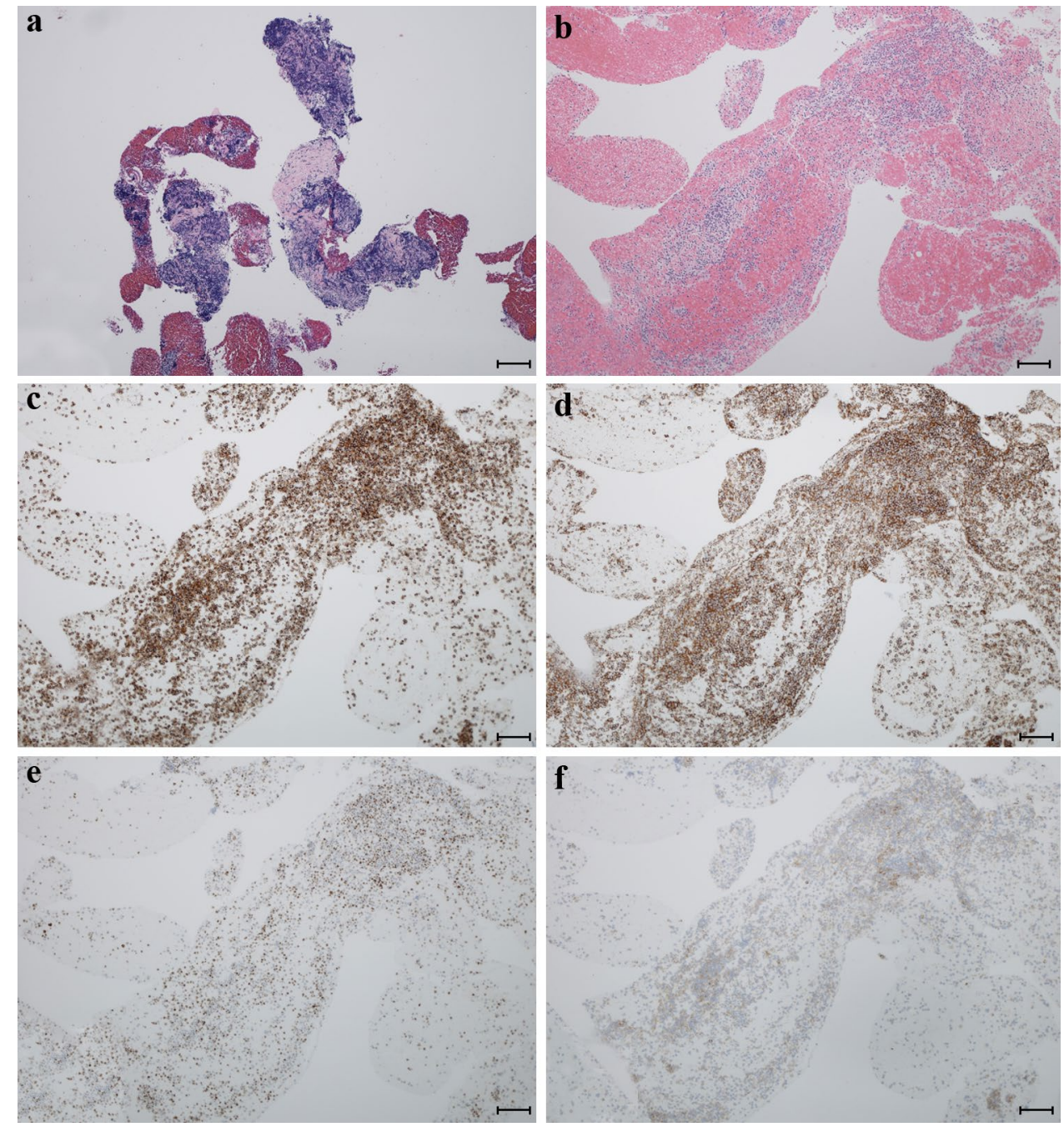

performed. Pathology was consistent with follicular thyroid cancer. Thus, the initial Lugano classification staging was determined to be stage IIE in retrospect. Maintenance obinutuzumab monotherapy was commenced thereafter, with no adverse effects other than one episode of neutropenia unaccompanied by fever. The patient remains in complete response 15 months after starting induction therapy.

\section{Case 2}

An asymptomatic 70-year-old man was referred for a pancreatic mass noted on screening abdominal ultrasound. He had a history of hypertension and cataract surgery. He had no family history of malignancies. Physical examination and laboratory tests including soluble interleukin-2 receptor, IgG4, CEA, and CA 19-9 were unremarkable. The hepatitis $\mathrm{B}$ panel was negative. CT with contrast revealed a slowlyenhancing mass in the pancreatic body and mildly enlarged mesenteric lymph nodes (Fig. 3a). FDG-PET/CT revealed minimally increased uptake (Fig. 3b).
EUS revealed a $15 \times 11 \mathrm{~mm}$ heterogeneous, clearly delineated hypoechoic mass in the pancreatic body with no dilation of the distal pancreatic duct. EUS-FNA was performed using a 22-gauge fine-needle biopsy needle (Acquire, Boston Scientific) (Fig. 4a). Two passes were made with suction and two passes were made using the slow-pull technique, with 20 strokes for each pass. Histological evaluation of the specimen revealed a monotonous proliferation of atypical lymphocytes without follicular architecture (Fig. 5a). Immunohistochemistry revealed that these cells were positive for BCL2, marginally positive for BCL6 and negative for CD10, but were indeterminate for lymphoma (Fig. 5b-d).

EUS-FNA was repeated after 3 months, when the lesion had grown to $16 \times 14 \mathrm{~mm}$ (Fig. 4b). This time, four passes were made with a 20-gauge EchoTip ProCore needle (Cook Medical, Bloomington, IN, USA). The same inconclusive findings were obtained, with negative immunnohistochemical staining for CD10. Another 3 months later, the pancreatic body mass had grown to $28 \mathrm{~mm}$ and a new, similar mass had appeared in the pancreatic tail (Fig. 4c, d). EUS-FNA 

tomography (CT) with contrast revealed a vaguely enhancing mass in the pancreatic body (arrow). b 18F-fluorodeoxyglucose positron emission tomography/CT revealed slightly increased uptake (arrow) in the pancreatic body
Fig. 3 a Initial computed
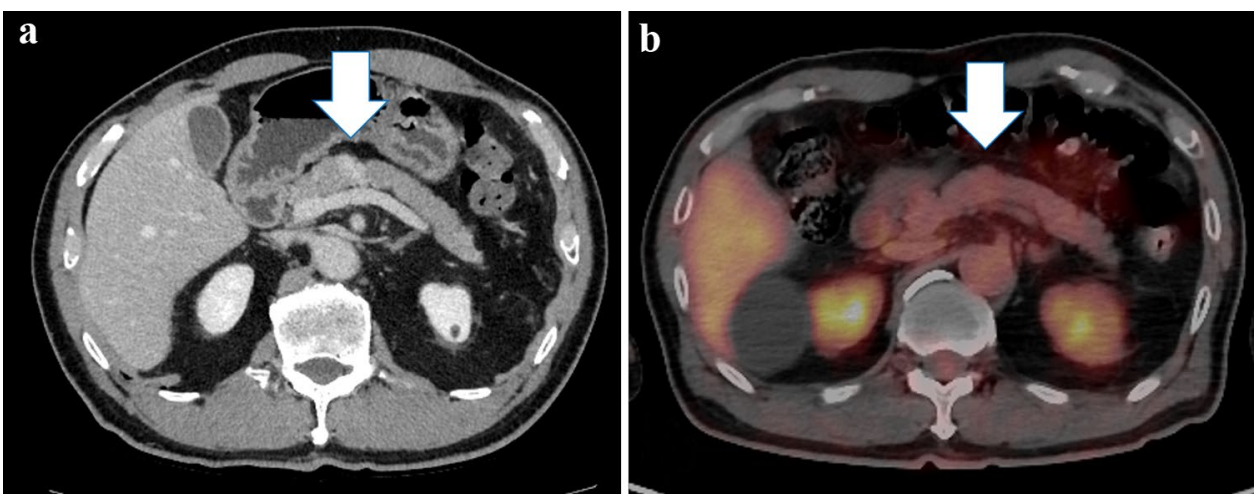

Fig. 4 a First endoscopic ultrasound-guided fine-needle aspiration of a $15 \times 11 \mathrm{~mm}$ heterogeneous, clearly delineated hypoechoic mass in the pancreatic body. b The mass had grown to $16 \times 14 \mathrm{~mm} 3$ months later. $\mathbf{c}$ The mass had grown to $28 \mathrm{~mm}$ another 3 months later. d A new, similar mass appeared in the pancreatic tail

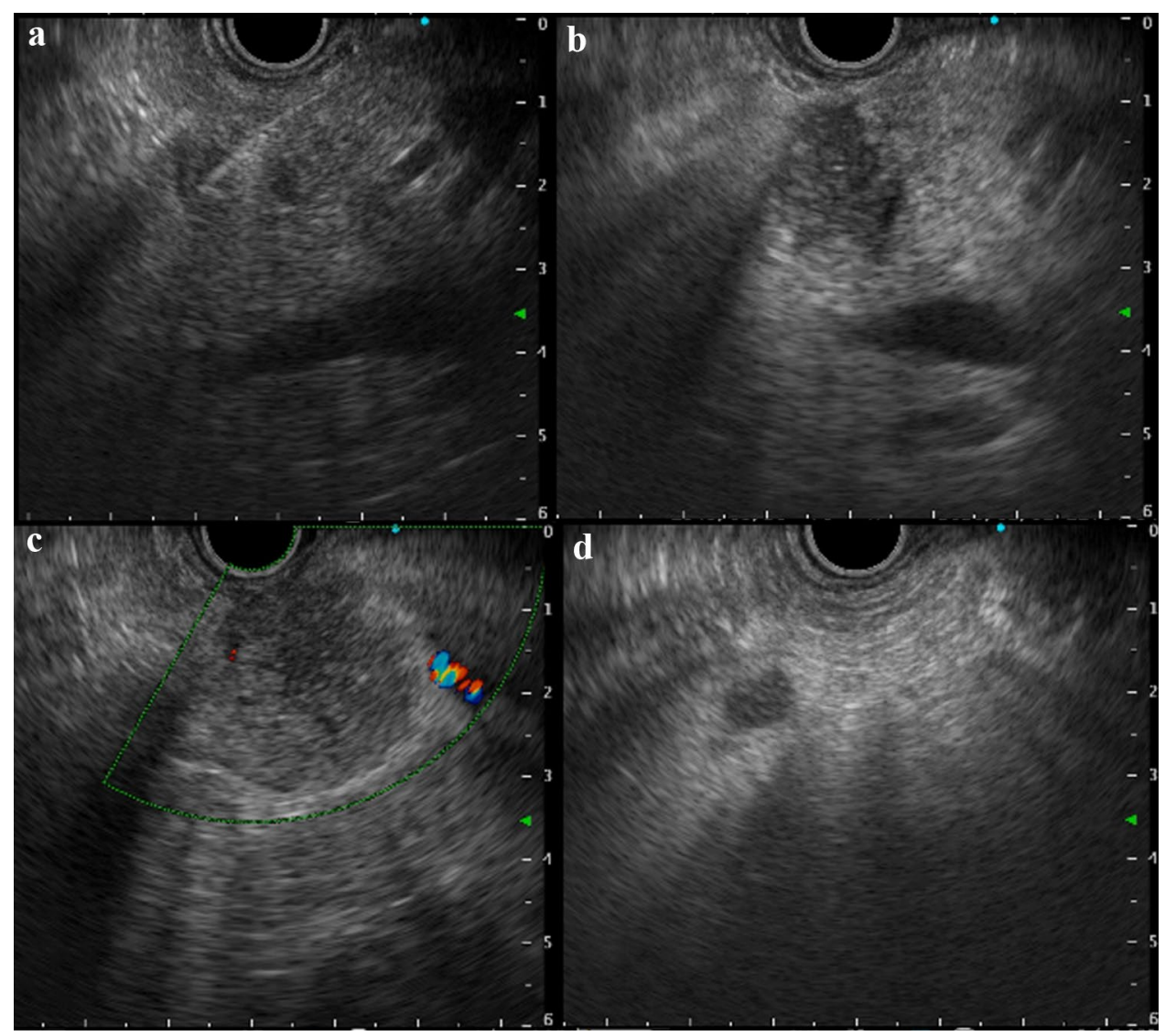

was repeated for both the pancreatic body mass and the new pancreatic tail mass, but a definite diagnosis could not be reached.

FDG-PET/CT taken after the third EUS-FNA session revealed increased uptake in the pancreas (SUVmax: 4.1) as well as abdominal, lower esophageal, and left supraclavicular lymph nodes. Consent for laparoscopic lymph node sampling was obtained and performed without complications. An abdominal lymph node measuring $21 \mathrm{~mm}$ was obtained. Histological evaluation revealed medium-sized atypical lymphocytes forming uniform and densely packed follicles. Immunohistochemistry was consistent with Grade 1-2 follicular lymphoma: CD5-, CD10 +, CD20 + . BCL2 +, BCL6 +, MUM1- (Fig. 6). Ki-67 labeling index was approximately $30 \%$. Flow cytometry also revealed a B-cell neoplasm $(\mathrm{CD} 19+, \mathrm{CD} 20+, \mathrm{CD} 10 \mathrm{dim}+)$ with lambda light chain restriction. Bone marrow aspiration was negative for malignancy. As a result, the patient was diagnosed with PPFL with low tumor burden, Grade 1-2, Lugano classification stage IIIE, FLIPI of 2 (age, stage): intermediate risk.

Although the tumor burden was low, the disease had progressed relatively quickly over a period of 6 months. Informed consent was therefore obtained for treatment with GB. Six cycles of GB led to complete response with 
Fig. 5 Histological evaluation of the first endoscopic ultrasound-guided fine-needle aspiration specimen (scale bars: $100 \mu \mathrm{m})$. a Hematoxylin and eosin stain revealed a dense, monotonous proliferation of atypical lymphocytes. Immunohistochemistry was positive for b BCL2, c marginally positive for BCL6, and $\mathbf{d}$ negative for CD10

Fig. 6 Histological evaluation of the surgically resected lymph node. a Hematoxylin and eosin stain revealed medium-sized atypical lymphocytes forming uniform and densely packed follicles (scale bar: $1 \mathrm{~mm}$ ). Immunohistochemistry was positive for b BCL2, $\mathbf{c}$ BCL6, and d CD10 (scale bars: $100 \mu \mathrm{m}$ )
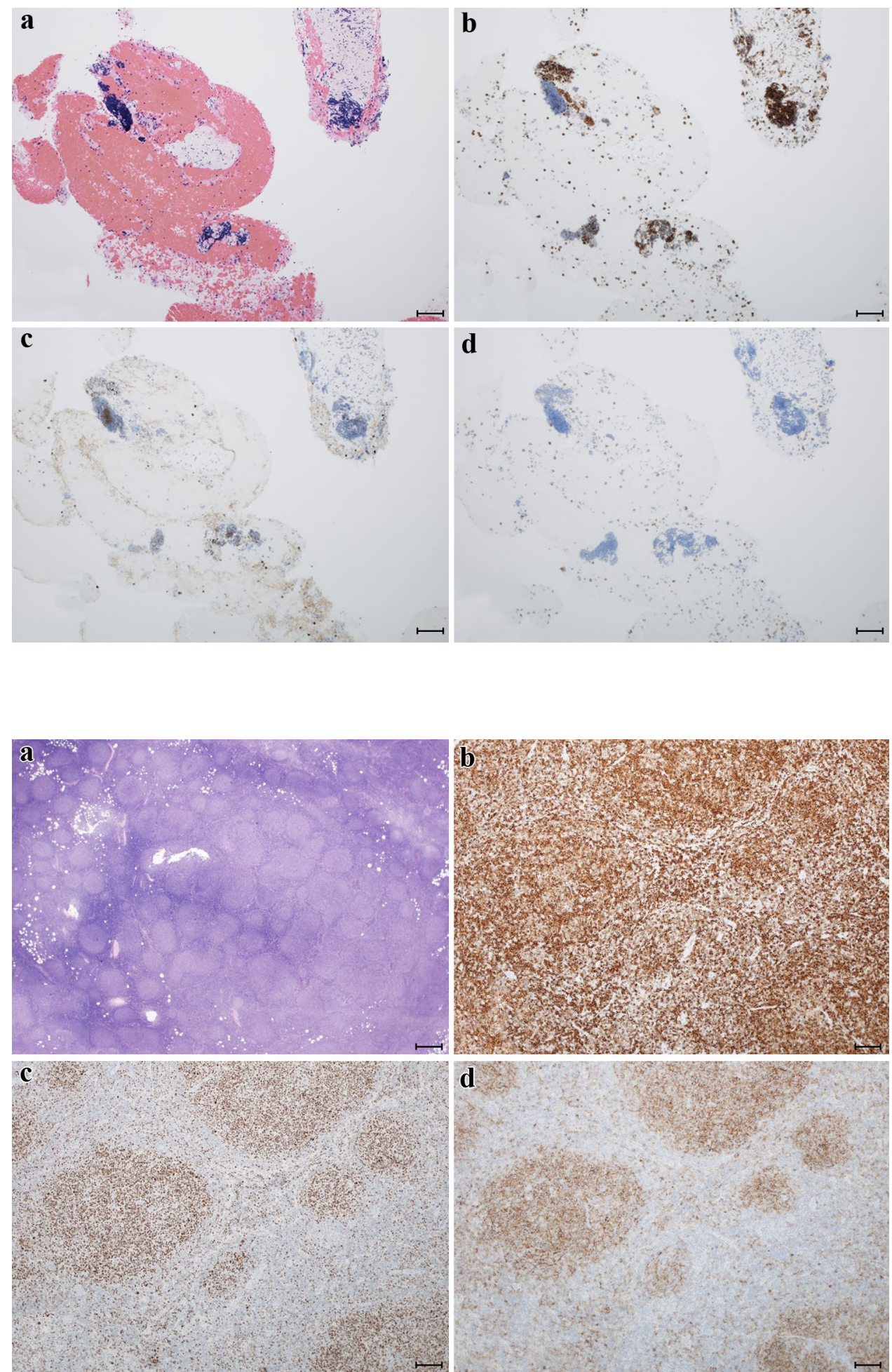

minimal adverse effects, which included one episode of Grade 3 neutropenia without fever. Although the patient opted for maintenance therapy, treatment was suspended 4 months later as the patient wished to receive the Severe Acute Respiratory Syndrome Coronavirus 2 vaccine [21].

\section{Discussion}

Lymphoma in the pancreas can take two forms: direct invasion from adjacent peripancreatic lymph nodes and, 
much less commonly, PPL. About $30 \%$ of patients with extranodal non-Hodgkin's lymphoma have pancreatic involvement [7]. On the other hand, the diagnosis of PPL can be made if lymphoma is mainly localized in the pancreas, even if there is peripheral lymph node involvement or distant spread [5]. The distinction between the two is therefore unclear. While both of our cases meet the criteria for PPFL, we cannot completely rule out the possibility that the lesions observed in Cases 1 and 2 arose from peripancreatic lymph nodes in the hepatoduodenal ligament and mesentery, respectively. Further research is required to determine differences in clinical characteristics and outcomes between the two entities.

Across the two largest PPL studies, the mean age at diagnosis was 57-63 years and 55-56\% were male [4, 19]. Most presented as large masses (mean $79-81 \mathrm{~mm}$ ). About $72 \%$ were located in the pancreatic head [4] while $10 \%$ presented as diffuse lesions [19]. Jaundice was observed in 33-47\% $[4,19]$. While $84 \%$ were diagnosed as early-stage disease, $67 \%$ were unresectable and $50 \%$ had lymphadenopathy [19]. Complete response was achieved with chemotherapy alone in $75 \%$ of cases. Endoscopic biopsy led to the diagnosis in $7-36 \%$ of cases, although it was unstated whether this involved EUS-FNA of the pancreatic lesion or endoscopic biopsies of duodenal invasion $[4,19]$.

Based on a review of the English literature over the last 30 years, we summarized the 33 reports of PPFL including our two cases in Table 1 [2, 4, 6-18]. Eighty percent of cases were male, with a median age of 67 years (range 51-82 years). While pancreatic head was involved in $87 \%$ of cases and median size was $70 \mathrm{~mm}$ (range 15-140 mm), jaundice was observed in only $25 \%$ of cases. Our cases represent the two smallest lesions reported to date and are the first cases to present with multiple pancreatic lesions. Excluding Case 2, all were discovered as Stage IE-IIE disease. While these characteristics are similar to PPL in general, PPFL may be more likely to affect males and less likely cause obstructive jaundice.

While imaging findings in PPL generally involve large, homogenous, hypovascular masses in the pancreatic head without pancreatic duct obstruction, differentiation from pancreatic ductal adenocarcinoma may be difficult [22]. EUS-FNA has become a standard diagnostic procedure for pancreatic masses, which may include suspected PPLs. According to one report, EUS-FNA of enlarged lymph nodes allowed for accurate diagnosis and classification of lymphomas in $88 \%$ of cases [23]. However, diagnostic yield of EUS-FNA for PPL remains low despite generally presenting as large masses [11]. In addition, EUS-FNA specimens cannot retain the follicular architecture which would aid in the diagnosis of PPFL.

While we successfully reached a diagnosis in Case 1 after a single EUS-FNA session, Case 2 could not be diagnosed despite obtaining satisfactory samples in 16 EUS-FNA passes during three different sessions over a course of 6 months. Diagnosis could not be reached in Case 2 despite using both Menghini needles and Franseen needles, both of which have high diagnostic yield [24, 25]. Repeated CD10 negativity was the key factor precluding the diagnosis of PPFL. Interestingly, the same phenomenon was observed in one previous report: EUS-FNA revealed CD19-positivity but CT-guided FNA was required to confirm CD10 expression [11]. Another report failed to reach the diagnosis despite performing EUS-FNA on the pancreatic lesion as well as multiple lymph nodes [14]. EUS-FNA was avoided in two other cases due to intervening vessels and hypervascularity, respectively $[15,16]$. Laparoscopic lymph node sampling should be considered in cases like these, where a definite diagnosis cannot be reached (1) on immunohistochemical grounds despite obtaining adequate FNA samples, (2) after multiple EUS-FNA attempts, or (3) because EUS-FNA cannot be performed safely.

In summary, EUS-FNA successfully diagnosed PPFL in only three of eight cases (38\%) where EUS-FNA was considered and in three of six cases (50\%) where EUS-FNA was actually performed. Difficulties in pathological diagnoses with EUS-FNA specimens have also been reported in diffuse large B-cell lymphoma: two cases reached a diagnosis only after the second EUS-FNA session and one case failed to reach a conclusive diagnosis despite finding atypical lymphocytes [26, 27]. According to one report, a higher number of EUS-FNA passes was required to diagnose PPFL compared to other PPLs [11].

The National Comprehensive Cancer Network Guidelines suggest anti-CD20 monoclonal antibody treatment with or without chemotherapy as an option for Grade 1-2 follicular lymphoma cases of all stages [28]. Watchful waiting is also an acceptable option for asymptomatic disease or when the tumor burden is low [29, 30]. Obinutuzumab, a new type II, glycoengineered, humanized anti-CD20 monoclonal antibody, demonstrated longer progression-free survival over rituximab and is now accepted as a part of standard first-line regimens for follicular lymphoma [20], including extranodal disease. Although its use in PFL has not been reported, we selected obinutuzumab-based regimens for our two cases based on the reported progression-free survival as well as the acceptable adverse events profile. Both patients also opted for maintenance obinutuzumab when suggested as an option to reduce relapse risk.

All reported PPFL cases in the literature were treated aggressively with surgery, chemotherapy, and/or radiotherapy except for one case when the patient refused treatment (and died of disease 6 years later) and another diagnosed only after autopsy $[4,10]$. Surgery may be selected when pre-operative diagnosis is inconclusive, for fear of possible pancreatic adenocarcinoma. Physicians may also be 


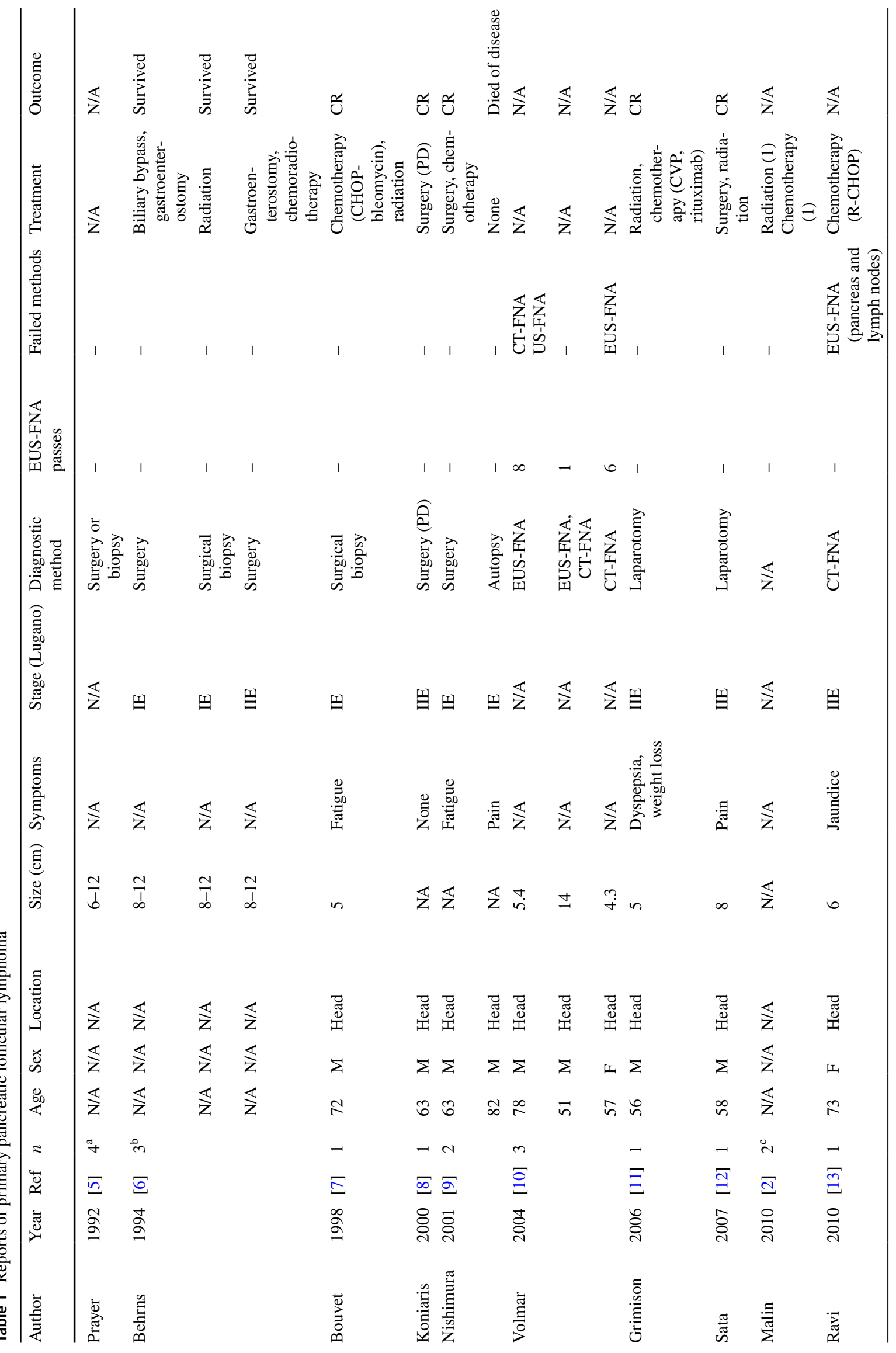




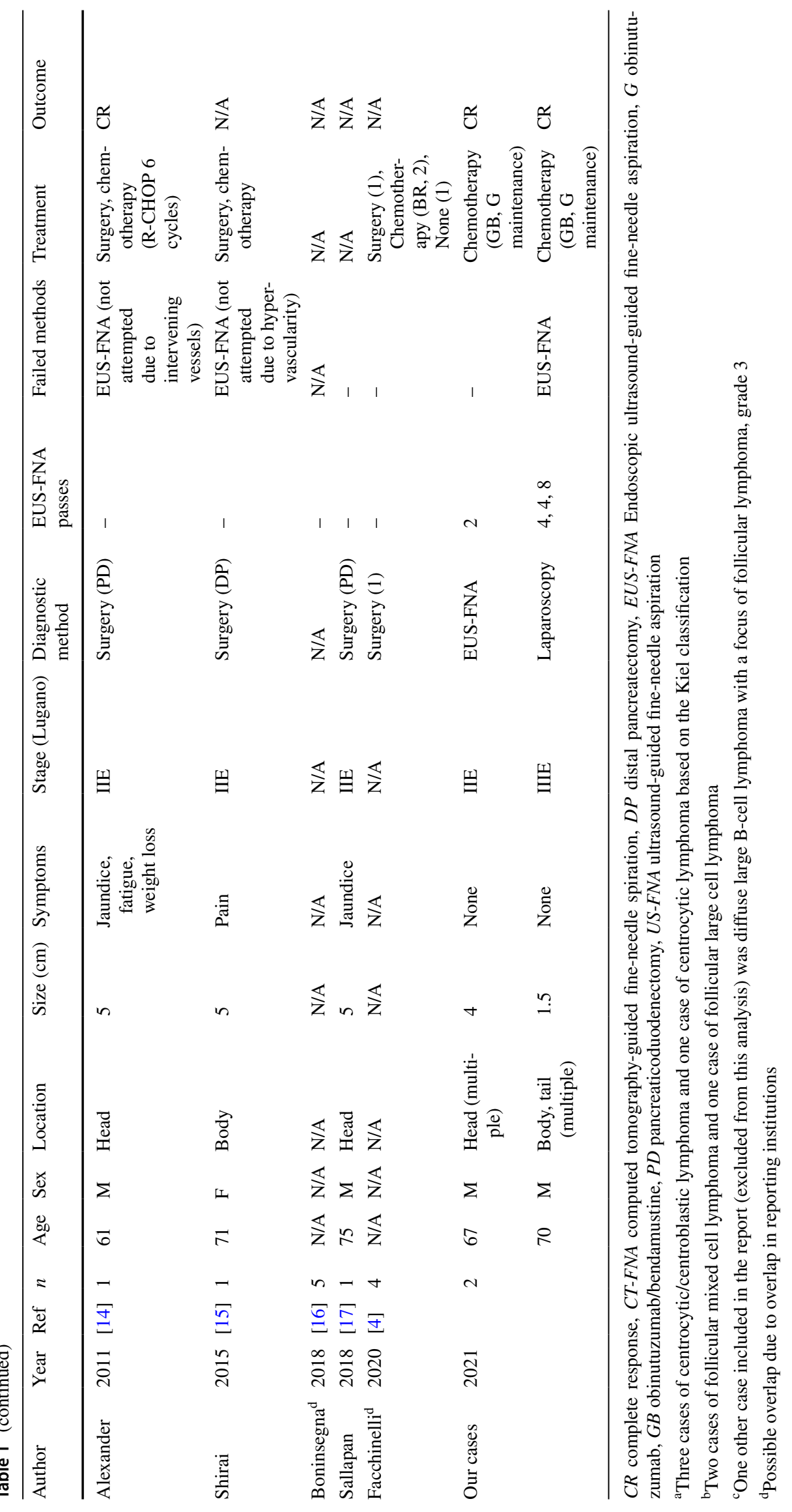


reluctant to leave PPFLs untreated as most occur in the pancreatic head, posing the risk of obstructive jaundice. Complete response has been reported in all cases treated by surgery, chemotherapy, radiotherapy, and combinations of these alternatives. While the literature appears to favor treatment over watchful waiting, the optimal approach remains unclear. Combination therapy used in some previous reports may be excessive treatment. It should also be noted that long-term follow-up is required as follicular lymphoma can relapse after an extended period of maintained response [31].

In conclusion, we report two cases of PPFL treated successfully with obinutuzumab and bendamustine induction therapy and maintained on obinutuzumab monotherapy. Pancreatectomy may be avoidable in advanced or multiple PPFL if a pre-operative diagnosis can be reached. Laparoscopic lymph node sampling may be beneficial when a diagnosis cannot be reached despite obtaining a satisfactory EUS-FNA specimen. Our literature review suggests a favorable prognosis after surgery, chemotherapy, and/or radiotherapy.

\section{Acknowledgments None.}

Author contributions TO wrote the manuscript. TS, NN, MT, CM, TM, TF, YY, TT, AK, MM, MO, DM, and NS critically reviewed the manuscript. All authors approved the final manuscript.

Funding The authors have received no funding for the publication of this article.

\section{Declarations}

Conflict of interest Takeshi Okamoto, Takashi Sasaki, Noriko Nishimura, Manabu Takamatsu, Chinatsu Mori, Takafumi Mie, Takaaki Furukawa, Yuto Yamada, Tsuyoshi Takeda, Akiyoshi Kasuga, Masato Matsuyama, Masato Ozaka, Dai Maruyama, and Naoki Sasahira declare that they have no conflict of interest.

Human Rights This study does not include any data about human subjects.

Informed Consent Informed consent was obtained from all patients for the publication of this case report.

\section{References}

1. Ezzat A, Jamshed A, Khafaga Y, et al. Primary pancreatic nonHodgkin's lymphomas. J Clin Gastroenterol. 1996;23:109-12.

2. Malin ES, Tomey CE, Ono J, et al. Primary pancreatic lymphoma. Blood. 2010;116:4150.

3. Baylor SM, Berg JW. Cross-classification and survival characteristics of 5000 cases of cancer of the pancreas. J Surg Oncol. 1973;5:335-58.

4. Facchinelli D, Sina S, Boninsegna E, et al. Primary pancreatic lymphoma: clinical presentation, diagnosis, treatment, and outcome. Eur J Haematol. 2020;105:468-75.

5. WHO Classification of Tumours Editorial Board. Digestive system tumors. Lyon (France): International Agency for Research on Cancer; 2019. (WHO classification of tumors series, 5th ed.; vol. 1): 376-407.

6. Prayer L, Schurawitzki H, Mallek R, et al. CT in pancreatic involvement of non-Hodgkin lymphoma. Acta Radiol. 1992;33:123-7.

7. Behrns KE, Sarr MG, Strickler JG. Pancreatic lymphoma: is it a surgical disease? Pancreas. 1994;9:662-7.

8. Bouvet M, Staerkel GA, Spitz FR, et al. Primary pancreatic lymphoma. Surgery. 1998;123:382-90.

9. Koniaris LG, Lillemoe KD, Yeo CJ, et al. Is there a role for surgical resection in the treatment of early-stage pancreatic lymphoma? J Am Coll Surg. 2000;190:319-30.

10. Nishimura R, Takakuwa T, Hoshida $Y$, et al. Primary pancreatic lymphoma: clinicopathological analysis of 19 cases from Japan and review of the literature. Oncology. 2001;60:322-9.

11. Volmar KE, Routbort MJ, Jones CK, et al. Primary pancreatic lymphoma evaluated by fine-needle aspiration: findings in 14 cases. Am J Clin Pathol. 2004;121:898-903.

12. Grimison PS, Chin MT, Harrison ML, et al. Primary pancreatic lymphoma-pancreatic tumours that are potentially curable without resection, a retrospective review of four cases. BMC Cancer. 2006;6:117.

13. Sata N, Kurogochi A, Endo K, et al. Follicular lymphoma of the pancreas: a case report and proposed new strategies for diagnosis and surgery of benign or low-grade malignant lesions of the head of the pancreas. JOP. 2007;8:44-9.

14. Ravi K, Sanchez W, Sweetser S. Primary pancreatic follicular lymphoma mimicking adenocarcinoma. Clin Gastroenterol Hepatol. 2010;8:e101-2.

15. Alexander RE, Nakeeb A, Sandrasegaran K, et al. Primary pancreatic follicle center-derived lymphoma masquerading as carcinoma. Gastroenterol Hepatol (N Y). 2011;7:834-8.

16. Shirai Y, Okamoto T, Kanehira M, et al. Pancreatic follicular lymphoma presenting as acute pancreatitis: report of a case. Int Surg. 2015;100:1078-83.

17. Boninsegna E, Zamboni GA, Facchinelli D, et al. CT imaging of primary pancreatic lymphoma: experience from three referral centres for pancreatic diseases. Insights Imaging. 2018;9:17-24.

18. Sallapan S, Abu Bakar NZ, Jarmin R, et al. Primary follicular lymphoma of the pancreas: a rare tumour mimicking pancreatic carcinoma. Malays J Pathol. 2018;40:359-71.

19. Sadot E, Yahalom J, Do RK, et al. Clinical features and outcome of primary pancreatic lymphoma. Ann Surg Oncol. 2015;22:1176-84.

20. Marcus R, Davies A, Ando K, et al. Obinutuzumab for the first-line treatment of follicular lymphoma. N Engl J Med. 2017;377:1331-44.

21. Tepasse PR, Hafezi W, Lutz M, et al. Persisting SARS-CoV-2 viraemia after rituximab therapy: two cases with fatal outcome and a review of the literature. Br J Haematol. 2020;190:185-8.

22. Anand D, Lall C, Bhosale $\mathrm{P}$, et al. Current update on primary pancreatic lymphoma. Abdom Radiol (NY). 2016;41:347-55.

23. Yasuda I, Tsurumi H, Omar S, et al. Endoscopic ultrasoundguided fine-needle aspiration biopsy for lymphadenopathy of unknown origin. Endoscopy. 2006;38:919-24.

24. Mie T, Sasaki T, Kanata R, et al. Comparison of endoscopic ultrasound-guided tissue acquisition using a 20-gauge Menghini needle with a lateral forward bevel and a 22-gauge Franseen needle a single-center large cohort study. Clin Endosc. 2021. https://doi.org/10.5946/ce.2020.251.

25. Mie T, Sasaki T, Kanata R, et al. Diagnostic yield of endoscopic ultrasound-guided tissue acquisition for small solid pancreatic lesions. Endosc Int Open. 2020;8:E1359-64.

26. Savari O, Al-Duwal Z, Wang Z, et al. Pancreatic lymphoma: a cytologic diagnosis challenge. Diagn Cytopathol. 2020;48:350-5. 
27. Kopel J, Swarup K, Thein K, et al. Primary B cell lymphoma of the pancreas. J Gastrointest Cancer. 2020;51(3):1077-80.

28. National Comprehensive Cancer Network: NCCN Clinical Practice Guidelines in Oncology (NCCN Guidelines®). B-cell Lymphomas Version 4.2021-May 5, 2021. Available from: https:// www.nccn.org/. Accessed 29 May 2021.

29. Ardeshna KM, Smith P, Norton A, et al. Long-term effect of a watch and wait policy versus immediate systemic treatment for asymptomatic advanced-stage non-Hodgkin lymphoma: a randomised controlled trial. Lancet. 2003;362:516-22.

30. Yuda S, Maruyama D, Maeshima AM, et al. Influence of the watch and wait strategy on clinical outcomes of patients with follicular lymphoma in the rituximab era. Ann Hematol. 2016;95:2017-22.
31. Manna M, Lee-Ying R, Davies G, et al. Autologous transplantation improves survival rates for follicular lymphoma patients who relapse within two years of chemoimmunotherapy: a multi-center retrospective analysis of consecutively treated patients in the real world. Leuk Lymphoma. 2019;60:133-41.

Publisher's Note Springer Nature remains neutral with regard to jurisdictional claims in published maps and institutional affiliations. 